http://www.jfas.info

\title{
WIRELESS REAL-TIME HAZE MONITORING DEVICE
}

\author{
N. Ya'acob*, A. L. Yusof, A. E. Azhar, N. F. Naim and M. S. M. Yusoff \\ Faculty of Electrical Engineering, Universiti Teknologi MARA, 40450 Shah Alam, Selangor, \\ Malaysia \\ Published online: 05 October 2017
}

\begin{abstract}
Typically, the information received by public is based on 24-hour PSI from pollutant $\mathrm{PM}_{10}$. The 24-hour PSI reading often cause some confusion as most of the time. It does not correlate visually with the environment as seen with naked eye. Inaccurate reading may bring serious effect to people with respiratory problem. Furthermore, the reading from pollutant $\mathrm{PM}_{10}$ is not accurate as it measures particle matter as small as 10 micrometer in diameter, while the harmful particle matter is smaller than that. This project proposes a real-time portable wireless haze monitoring device, based on pollutant $\mathrm{PM}_{2.5}$ to measure air quality and beaglebone black controller to control the transmission process of measured data taken via wireless data network to the MySQL database. The reading, which is real-time reading and more accurate compared to pollutant $\mathrm{PM}_{10}$, can provide haze awareness and guidance to people to plan their activities.
\end{abstract}

Keywords: PSI; PM10; PM2.5; beaglebone black.

Author Correspondence, e-mail: norsuzilabintiyaacob@yahoo.com

doi: http://dx.doi.org/10.4314/jfas.v9i4s.29

\section{INTRODUCTION}

Haze is an atmospheric phenomenon where dust, smoke and other dry particles, obscure the clarity of the sky [1]. It also contains six air pollutants such as particulate matter, nitrogen dioxide, sulphur dioxide, ozone and carbon monoxide. Particulate matter (PM) is the term 
used to define a mixture of solid particles and liquid droplets which exist in the air. These particles come in many shapes and sizes namely 2.5 micrometer and 10 micrometer and are mostly the result of illegal burning of forests. Smaller particles can enter deep into the lungs and in some cases, it can enter into a person's bloodstream, resulting irritation to the eye, nose and throat. More damaging effect can be seen on a person with respiratory problem, whereby the risk of getting asthma attacks and heart attacks or heart failure is high.

Wireless network is defined as any type of computer network that uses wireless data connections for connecting network nodes [2]. Wireless networking is a method by which homes, telecommunications networks and business enterprises avoid the costly process of installing cables into a building, or as a connection between various equipment locations [3]. Wireless telecommunications networks uses radio communication to communicate from the transmitter to the receiver. One of the applications of wireless communication is wireless sensor network (WSN). In WSN, each sensor node is able to perform some processing and sensing tasks independently. The usual hardware components required to construct a sensor node include radio transceiver, embedded processor, internal and external memories, power source and sensor(s) [4].

From previous research [5], it is stated that Beta Attenuation Monitoring (BAM) is selected to be the standard scheme used by Department of Environment (DoE) to measure particulate matter in 52 monitoring stations in Malaysia. The system is based on 24-hour API reading, which means the reading is given post 24 hours of measurement.

This project proposes a system that provides real-time API value which means the current up-to-date API value of a time at a particular place and is able to retrieve data several meters away without contact and line-of-sight, therefore reducing the need for manual reading [6]. This system proposes to send data wirelessly using data network. The system starts operating when the pollutant detects particles and transmit the reading value to the database continuously.

This device is designed to be portable in order to make it more user friendly. It uses beaglebone black as its main controller. The beaglebone black board consists of sierra wireless which is used as a wireless medium to send data. In addition to that, it is connected to Global Positioning System (GPS) to navigate the coordinates of sensor. A Shinyei PDP42NS dust sensor is used to detect dust and particular matter of 2.5 micrometer and above in diameter. All the components used in this device are compact, making it mobile. 
The objective of this project is to develop a portable, wireless haze monitoring device which can be used to measure haze anywhere and anytime. Built with more accurate pollutant namely PM2.5, it can measure small particle matters up to 2.5 micrometer in size. The device is designed to provide real-time reading of haze to users and thus inform them of the current haze situation.

\section{METHODOLOGY}

This research is divided into two parts; hardware and software development. The overall system design for the hardware consists of three main works which are data collections, data processing and data communication. The components which make up wireless haze monitoring system are PM sensor, GPS module, beaglebone black microcontroller and MySQL database.

The overall system design as shown in Fig. 1 will include data collection, data communication, data integration and analysis. The real-time data collection system is represented by $\mathrm{PM}_{2.5}$ dust sensor and GPS module. Data communication involves wireless sensor network devices and client-server protocol to send collected data. Beaglebone black microcontroller is used to manage data collection and send them to MySQL database. All sensor data from the database and other haze related information will be used for display at the website and can be used for analysis and forecasting study.

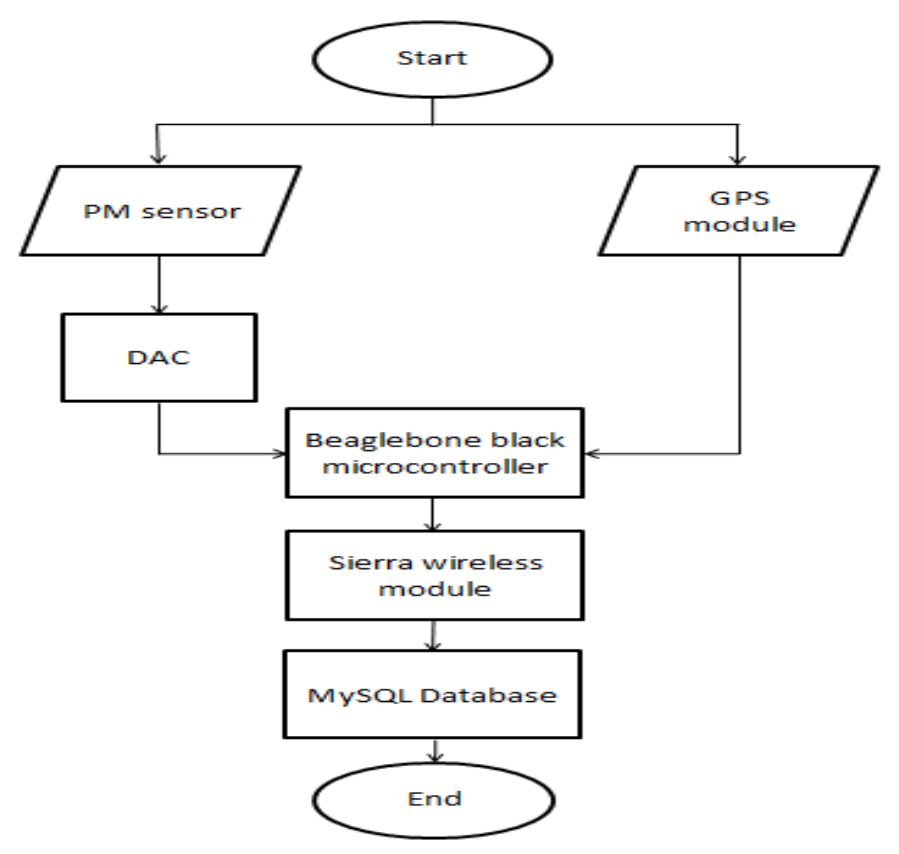

Fig.1. Overall haze monitoring system flow chart 


\subsection{Hardware Development}

\subsubsection{Sensor}

PM sensor Shinyei PPD42NS is used to detect particulate matter of 2.5 micrometer and above in size. This sensor functions by transmitting digital output (Lo Pulse) to particulate matters. Lo Pulse Occupancy time (LPO time) is recorded in proportion to particulate matter concentration [7]. This sensor operates by converting up the particles and then sends a light beam provided by the infrared LED. Light scattered by particles at a forward angle of about $45^{\circ}$ is picked up by photodiode. The light path is baffled to avoid stray light pickup, and a lens in front of the photodiode focuses into a detection region in the air flow and close to the LED light portal. JCA-001 GPS active antenna is used to track the location of the haze monitoring system directly from satellite. The value received is in terms of latitude and longitude. Digital to analogue converter (DAC) is designed to convert digital value from $\mathrm{PM}_{2.5}$ dust sensor into analogue value.

\subsubsection{Beaglebone Black Microcontroller}

Beaglebone black (BBB) as depicted in Fig. 2 acts as the mind for this haze monitoring system. This Texas Instrument AM335x1GHz ARM Cortex-A8 Processor functions as a microcontroller to control the input received from sensors and control their outputs. BBB is an advanced microcontroller, which is low-cost, open source and community-supported development platform for ARM Cortex-A8 processor developers.

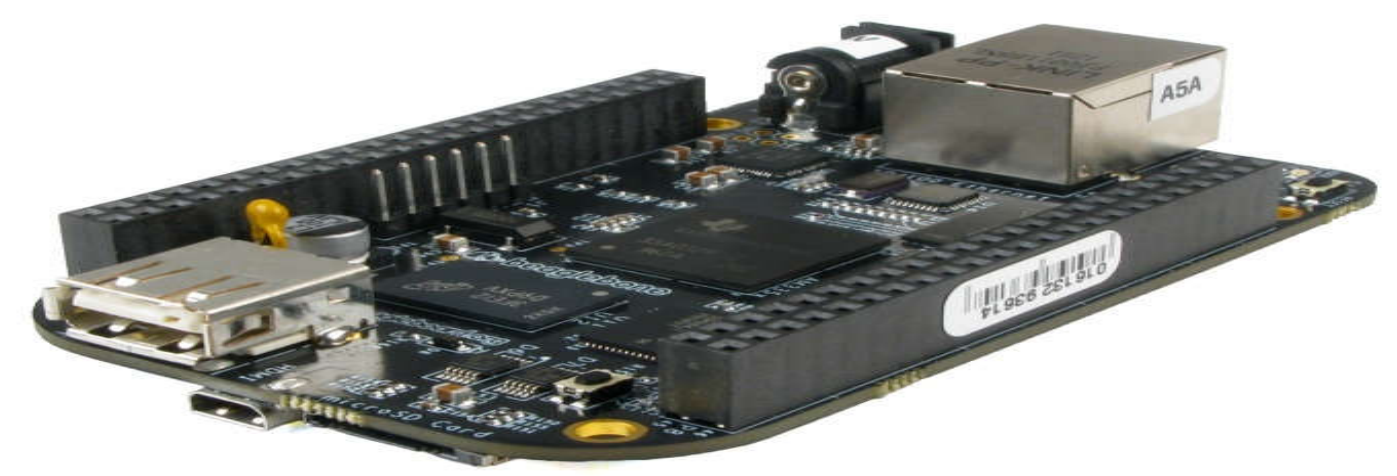

Fig.2. Beaglebone black controller

\subsubsection{Sierra Wireless Module}

Sierra Wireless MC7304 is used in this project as it supports $3 \mathrm{G}$ and $4 \mathrm{G}$ global networks including LTE Advanced with automatic fallback to $3 \mathrm{G}$ and $2 \mathrm{G}$ networks, depending on the signal availability. It is used to send data retrieved from BBB to the built-in database at the 
cloud. GNSS tracking, which ist available in this chipset supports the JCA-001 GPS active antenna to locate the position of the completed hardware.

\subsubsection{MySQL Database}

MySQL database is an open-source relational database management system [8]. It is used to store and retrieve data received from BBB. A table is created in this MySQL database to store the data.

\subsection{Software Development}

Fig. 3 shows the flow chart of how BBB system works. BBB is programmed to receive data from all sensors and GPS antenna, process and transmit them into a database [9-10]. MySQL database is used to record all the data collected based on real time value coming from both sensors. The data is then processed and displayed at the website and application in terms of Air Pollution Index (API) scale. The process of reading and sending data continuously updated until the system is put to stop or halt.

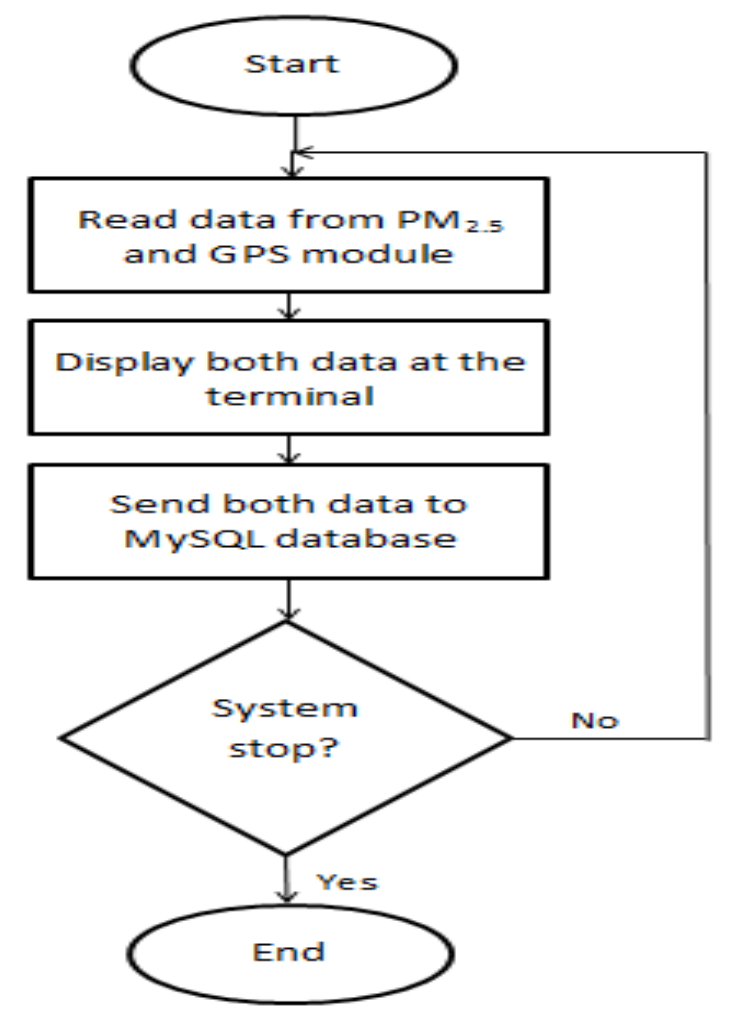

Fig.3. Flow chart of beaglebone black microcontroller

BBB requires Ubuntu which is a Debian-based Linux Operating System (OS) to act as another machine to develop and build the project. Software program called Eclipse IDE is a cross-compiler in Linux OS which makes processors compatible with ARM architecture [11]. 
Fig. 4 shows the Eclipse IDE running in Ubuntu.

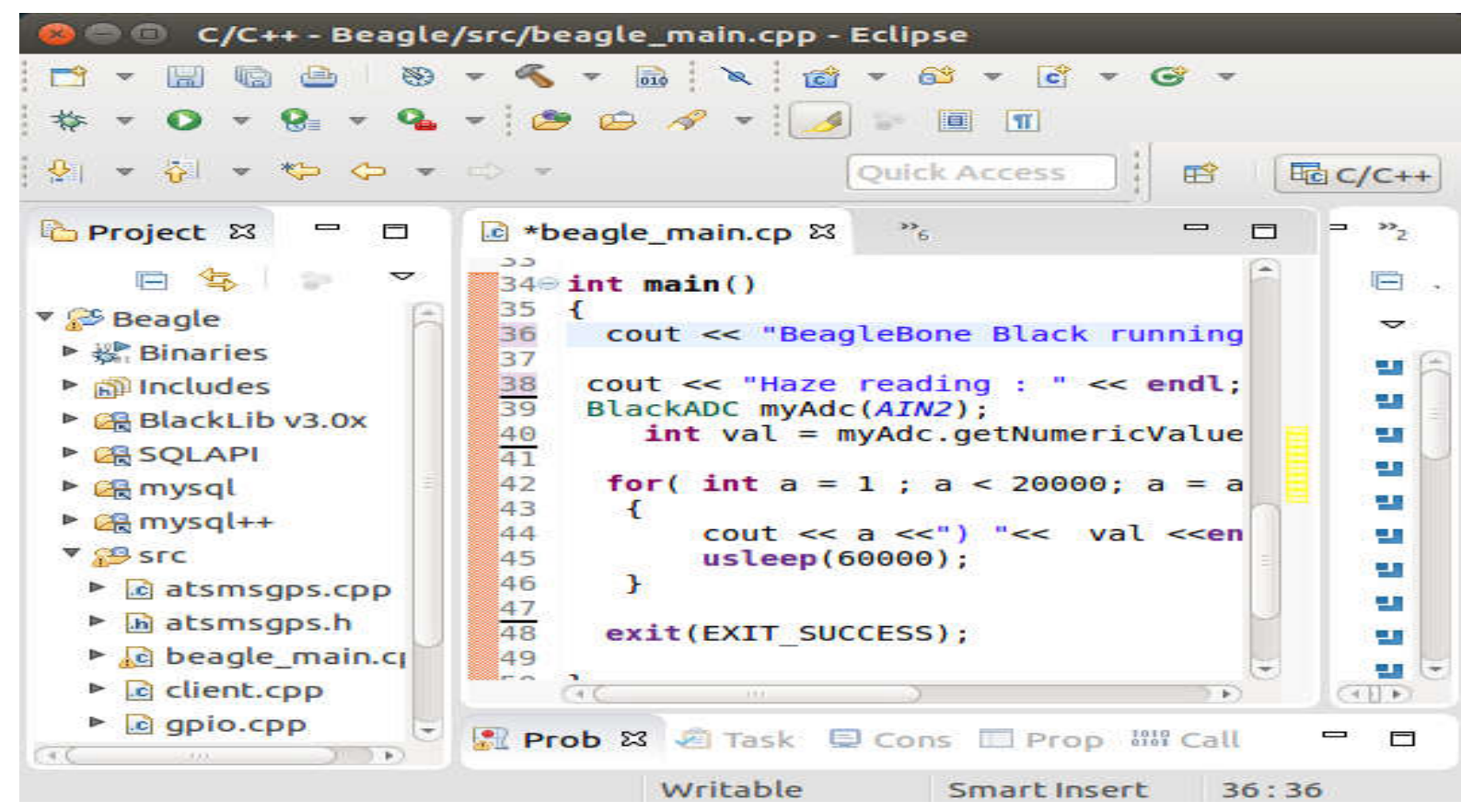

Fig.4. Eclipse Software installed through the Ubuntu Operating System

\section{RESULT AND DISCUSSION}

All the components are set up as shown in Fig. 5. Sections labelled with A, B, C, D and E are the BBB, Sierra MC7304 Wireless module, Shinyei PPD42NS, digital-to-analog converter and GPS antenna respectively.

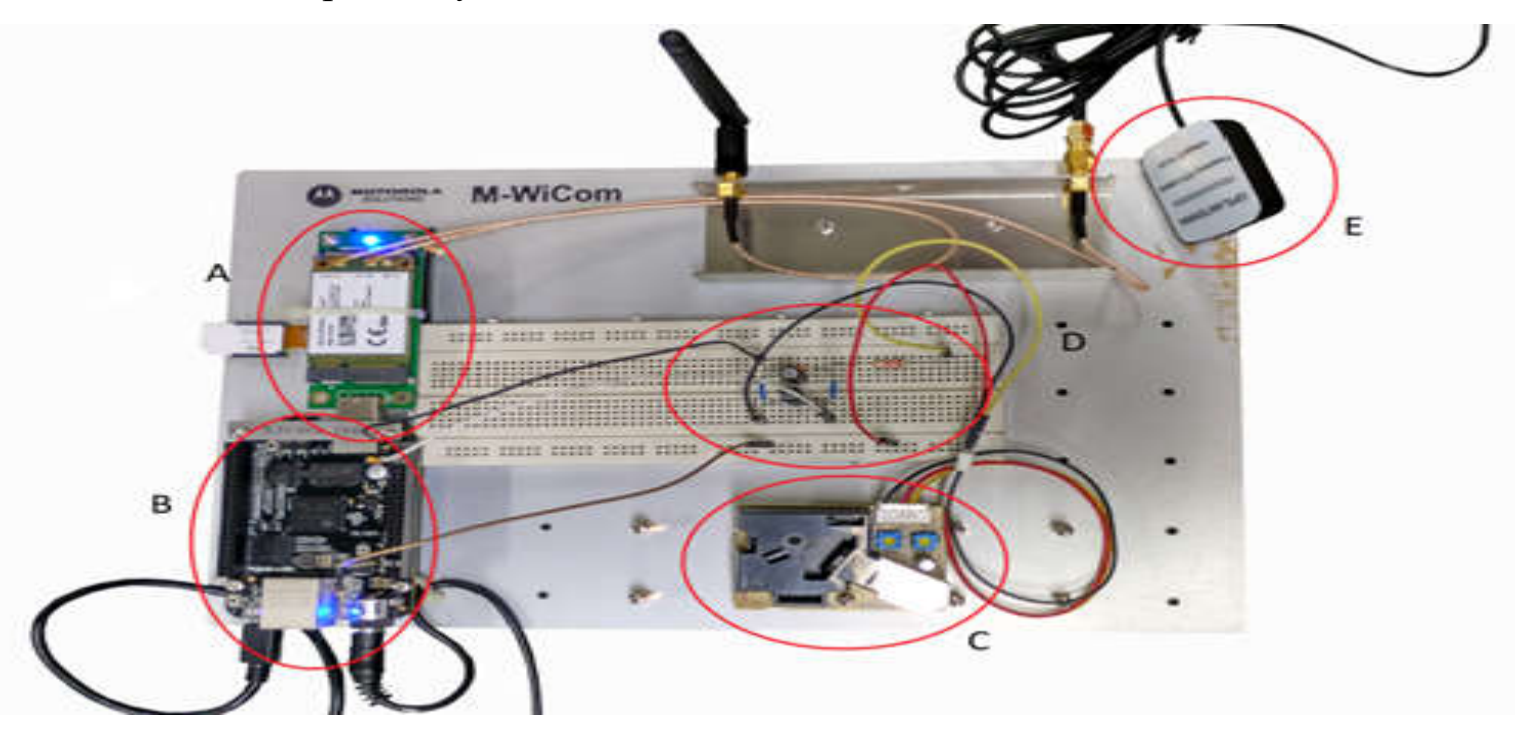

Fig.5. Complete hardware setup

Pin 3 from the Shinyei PPD42NS is connected to the 5V pin at P9 header of BBB as supply voltage to operate the sensor. The output of the sensor, Pin 2 is connected to DAC before 
being connect to the BBB. This is because BBB can only receive input that is below $3.3 \mathrm{~V}$. DAC is designed to convert the $5 \mathrm{~V}$ digital signal from the sensor into $3.3 \mathrm{~V}$ analog signal. Overload input voltage can make the BBB short-circuited and cause damage to the board. The output of DAC is then connected to AIN0 (analog input pin 0) at the P9 header of the BBB. GPS antenna is connected to the sierra wireless module using SMB connector. Dipole antenna is attached to increase signal strength of the sierra wireless module. Sierra wireless module has one sim card connected to it. This sierra wireless is connected with BBB using USB port. Sierra wireless module uses $3 \mathrm{G}$ or Long Term Evolution (LTE) network provided by the sim card to send data collected.

Shah Alam, a city in the state of Selangor in Malaysia is selected as the location of measurement because of the large population of people and lack of forests. Data collected from sensors and GPS antenna is transmitted and arranged into the database. Fig. 6 shows the status of data being processed by the BBB, displayed at the terminal of Ubuntu. Data collection is taken per minute and then it is processed to display the average of 15 minutes haze readings to the database. The reading is taken for six hours at the selected location.

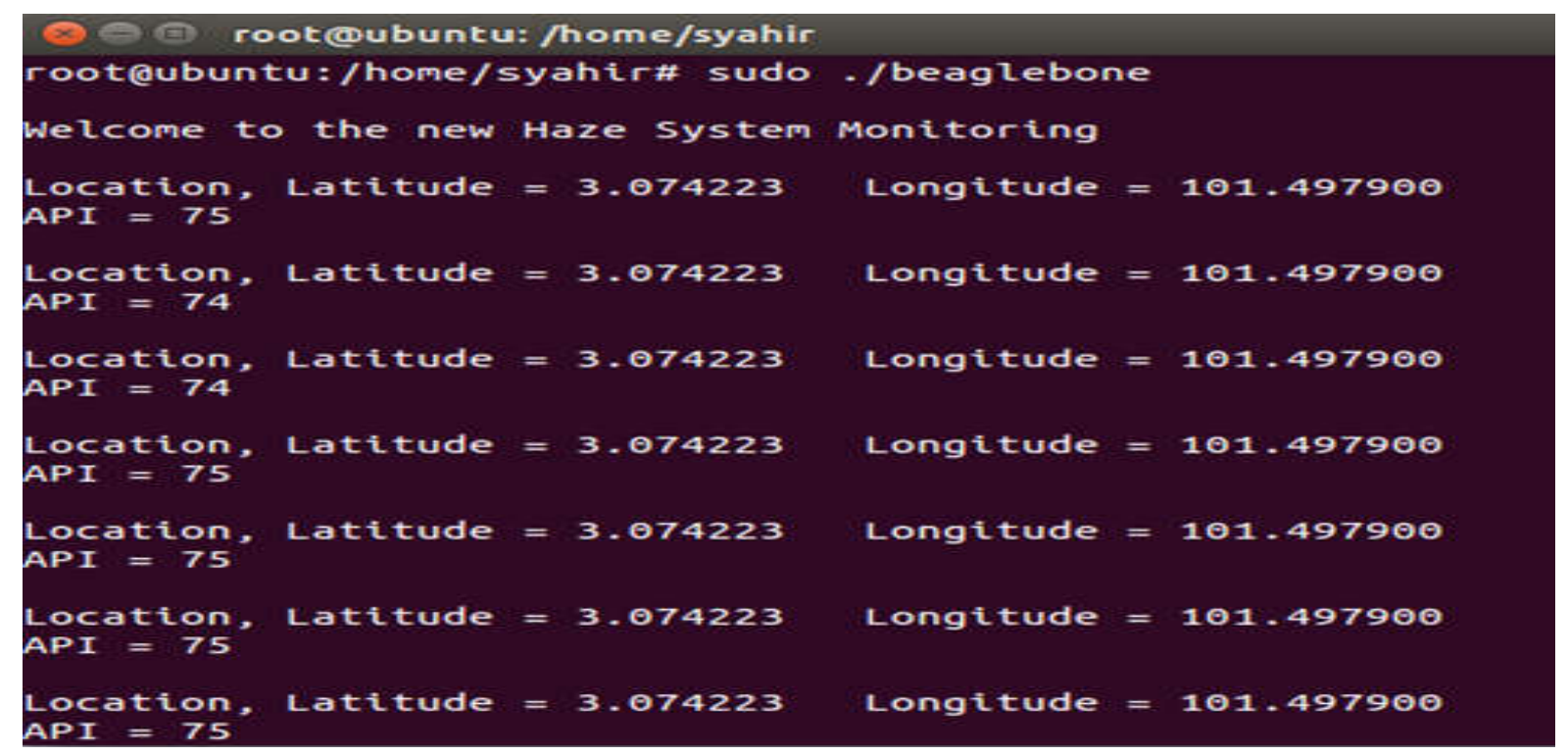

Fig.6. Data display at the Ubuntu terminal

The location in Fig. 6 is based on latitude and longitude value, which points to UiTM Shah Alam and API refers to the level of haze. The API value follows the standard of API for $\mathrm{PM}_{2.5}$ as show in Table 1. The API of 75 shown in Fig. 6 is categorized as moderate. Its mean the level of haze is normal and not dangerous for normal healthy person. 
Table 1. API standard for $\mathrm{PM}_{2.5}$

\begin{tabular}{|c|c|}
\hline Index Value & AQI Category \\
\hline $0-50$ & Good \\
\hline $51-100$ & Moderate \\
\hline $101-150$ & Unhealthy for Sensitive Groups \\
\hline $151-200$ & Unhealthy \\
\hline $201-300$ & Very Unhealthy \\
\hline $401-500$ & Hazardous \\
\hline
\end{tabular}

The haze data collected is then transmitted to the MySQL database using data network. Fig. 7 shows the data being stored in the database. There are four tables in the database for storing the data collected such as time, latitude, longitude and API.

MySQL database is programmed to receive readings of latitude, longitude and API value for every 15 minutes. The 3.074223 latitude and 101.487900 longitude value show constant value because it is setup and tested at the same location where API is measured. During the 5 hours recordings of data, it is shown that the highest API starts occuring at 2.15PM and its value is 81. Based on the API records in the MySQL database, the API during the 5 hours is considered as moderate level. This MySQL database can be displayed at website to give information about haze to users.

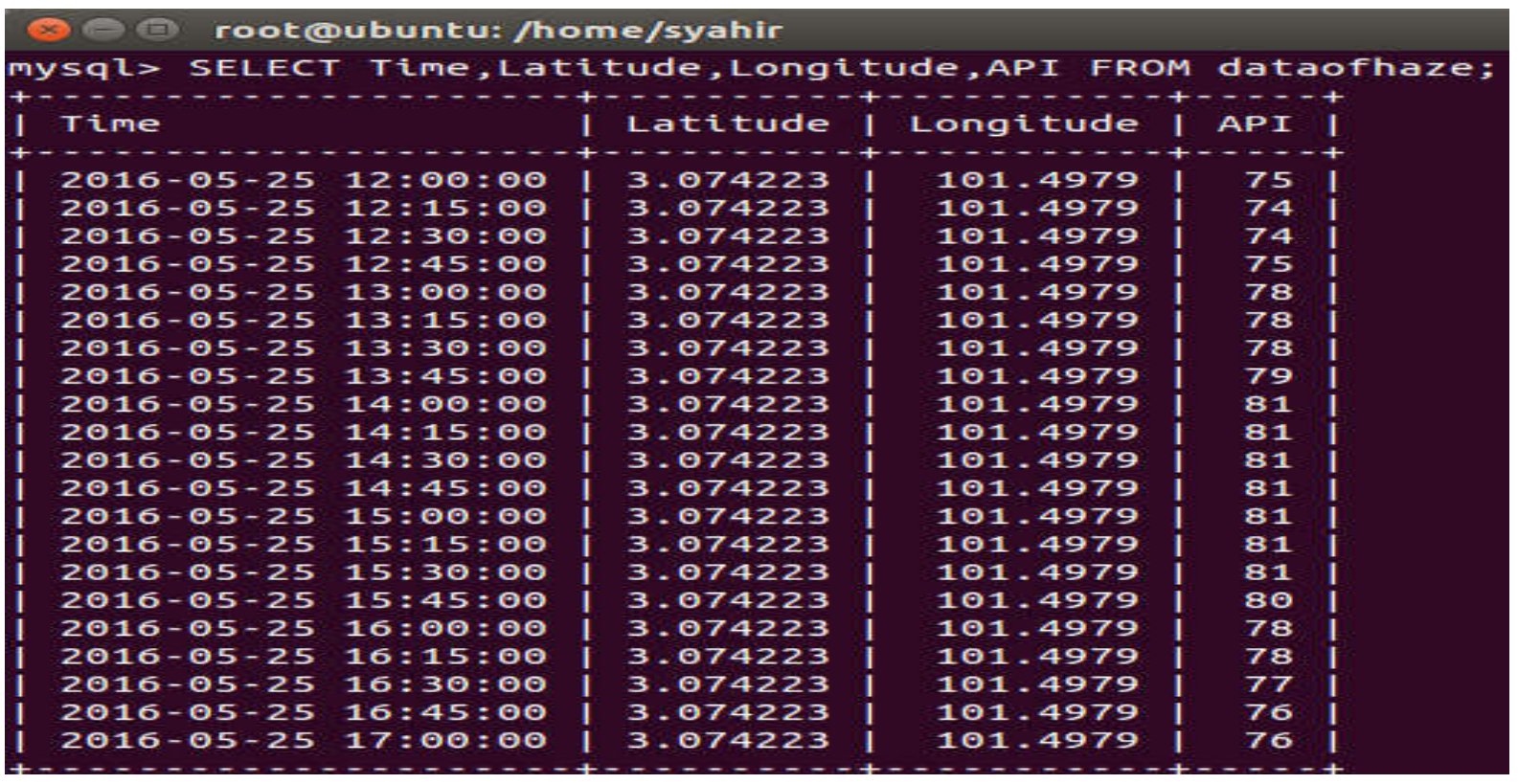

Fig.7. MySQL database table 


\begin{tabular}{|l|c|c|c|c|c|c|}
\hline $\mathrm{API}$ & $12.00 \mathrm{PM}$ & $01.00 \mathrm{PM}$ & $02.00 \mathrm{PM}$ & $03.00 \mathrm{PM}$ & $04.00 \mathrm{PM}$ & $05.00 \mathrm{PM}$ \\
\hline $\begin{array}{l}\text { Actual API Reading } \\
(\mathrm{PM}\end{array}$ & 46 & 50 & 58 & 58 & 50 & 48 \\
\hline $\begin{array}{l}\text { Experiment Reading } \\
\left(\mathrm{PM}_{2.5}\right)\end{array}$ & 75 & 78 & 81 & 81 & 78 & 76 \\
\hline \begin{tabular}{l} 
Percentage Error (\%) \\
\hline
\end{tabular} & 63.04 & 56.00 & 39.66 & 39.66 & 56.00 & 58.33 \\
\hline
\end{tabular}

Fig.8. Tabulation of actual and experimental result

For the result comparison, actual data is retrieved from Department of Environment (DoE)'s websit. Fig. 8 shows the actual and experimental data taken from noon until 5.00 PM. Actual API reading is the collected data from BAM of DoE and the experiment reading is the collected data from sensor at the BBB. The results obtained are compared to get percentage error. The highest percentage error, which is $63.04 \%$, occurs at $12.00 \mathrm{PM}$ due to the fact that actual API reading uses PM10 while experiment reading uses PM25. This proves that PM10 can only measure big particles compared to PM25, which measures smaller particles that are present in the air.

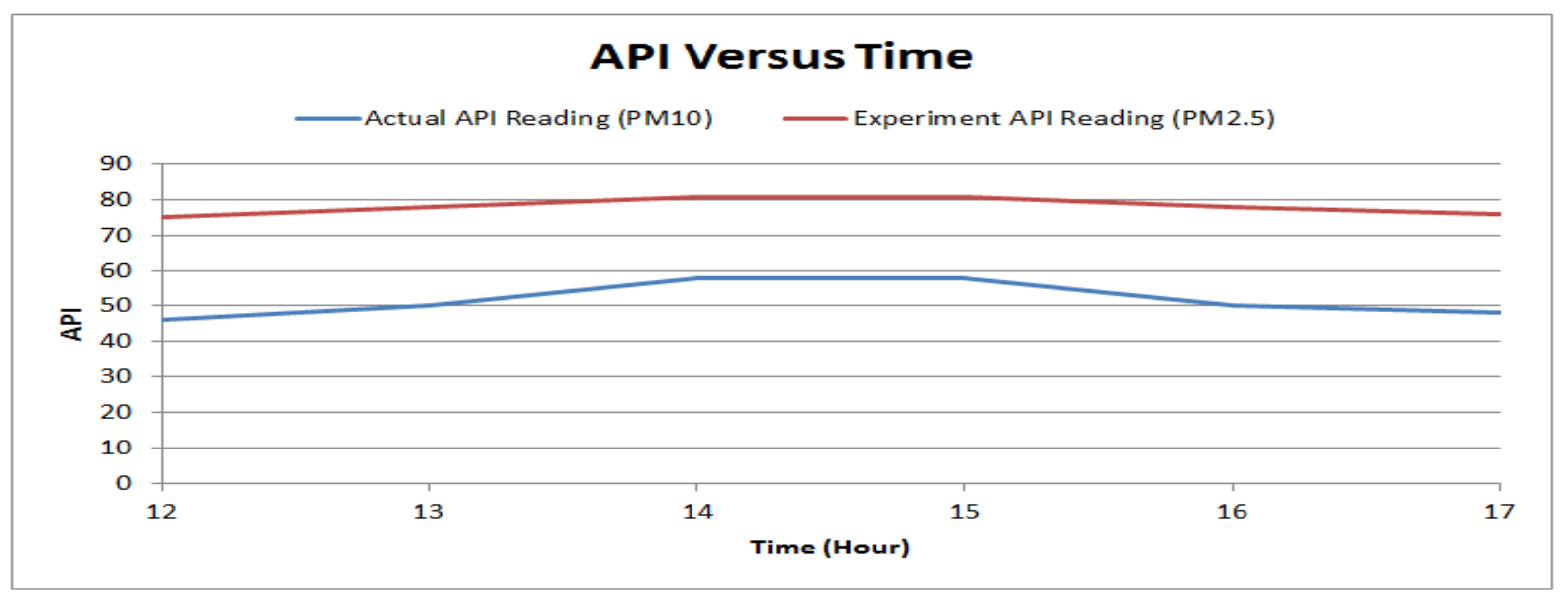

Fig.9. Graph of actual API and experiment reading

Fig. 9 compares API data collected from BBB and actual data retrieved from DOE. The level of experiment API reading is fairly higher compared to the actual API reading. This is because DoE use PM10 sensor that can detect particulate as small as 10 micrometer, while BBB use PM2.5 to get more accurate data of smaller particles present in the air.

\section{CONCLUSION}

This paper presents an advanced haze monitoring system with the latest technology. With the implementation of beaglebone black microcontroller, data and GPS coordinate collected from 
sensors are sent automatically to MySQL database via data network. The data is then processed before being displayed to any device. This monitoring system is convenient to people because the data in the database can be displayed as real-time result of haze using web interface and phone application. Therefore, people can plan and change their activities in that day according to the reading of haze. Besides real-time monitoring system, the reading is far more accurate as it can measure particle matter with diameter of 2.5 micrometer and above compared to the previous technology which only can measure the particle matter above 10 micrometre. It is desgined to be low power, affordable and portable to monitor their surrounding condition. For future recommendation, the system can be improved by adding more devices or nodes to get more precise data to cover wider area. Adding a camera is also recommended to capture the current situation.

\section{ACKNOWLEDGMENTS}

The authors would like to thank Faculty of Electrical Engineering, Universiti Teknologi MARA (UiTM) for their valuable support. This research is partly funded by the Malaysian Government through UiTM under 600-RMI/DANA5/3/REI (1/2015).

\section{REFERENCES}

[1] Othman J, Sahani M, Mahmud M, Ahmad M K. Transboundary smoke haze pollution in Malaysia: Inpatient health impacts and economic valuation. Environmental Pollution, 2014, 189:194-201

[2] Miao G., Zander J., Sung K. W., Slimane S. B. Fundamentals of mobile data networks. England: Cambridge University Press, 2016

[3] Andrea G. Wireless communications. England: Cambridge University Press, 2012

[4] Zheng J., Jamalipour A. Wireless sensor networks: a networking perspective. New Jersey: John Wiley and Sons, 2009

[5] Yusof N F, Ramli N A, Yahaya A S, Sansuddin N, Ghazali N A, al Madhoun W. Monsoonal differences and probability distribution of PM10 concentration. Environmental Monitoring and Assessment, 2010, 163(1-4):655-667 
[6] Othman K A, Abdullah E H, Li N, Rashid N E. GSM wireless technology implementation in haze monitoring. In 4th IEEE International Conference on Digital Information and Communication Technology and it's Applications, 2014, pp. 368-371

[7] Tracy A. De-construction of the Shinyei PPD42NS dust sensor (version 0.2). California: EME Systems, 2013

[8] Steven J V N. WebScaleSQL: MySQL for Facebook-sized databases. London: ZDNet, 2014

[9] Lextrait, V. The programming languages Beacon (v16). 2016, http://www.lextrait.com/vincent/implementations.html

[10] Kumar D K, Muralimohan M N, Saidulu S. GSM wireless technology implementation in HAZE Monitoring. International Journal of Ethics in Engineering \& Management Education, 3(7):7-10

[11] Lars V. Eclipse IDE tutorial. http://www.draelshafee.net/spring2011/Eclipse-tutorial.pdf

How to cite this article:

Ya'acob N, Yusof AL, Azhar AE, Naim NF, Yusoff MSM.Wireless real-time haze monitoring device. J. Fundam. Appl. Sci., 2017, 9(4S), 507-517. 